\title{
Genome-wide association study identifies a major gene for beech bark disease resistance in American beech (Fagus grandifolia Ehrh.)
}

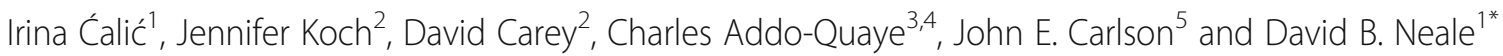

\begin{abstract}
Background: The American Beech tree (Fagus grandifolia Ehrh.), native to eastern North America, is ecologically important and provides high quality wood products. This species is susceptible to beech bark disease (BBD) and is facing high rates of mortality in North America. The disease occurs from an interaction between the woolly beech scale insect (Cryptococcus fagisuga), one of two species of the fungus Neonectria ( $N$. faginata or $N$. ditissima), and American Beech trees.
\end{abstract}

Methods: In this case-control genome-wide association study (GWAS), we tested $16 \mathrm{~K}$ high quality SNPs using the Affymetrix Axiom $1.5 \mathrm{~K}-50 \mathrm{~K}$ assay to genotype an association population of 514 individuals. We also conducted linkage analysis in a full-sib family of 115 individuals. Fisher's exact test and logistic regression tests were performed to test associations between SNPs and phenotypes.

Results: Association tests revealed four highly significant SNPs on chromosome (Chr) 5 for a single gene (Mt), which encodes a mRNA for metallothionein-like protein (metal ion binding) in Fagus sylvatica. Metallothioneins represent Cys-rich metal chelators able to coordinate metal atoms and may play an important role in the resistance mechanisms against beech scale insect.

Conclusion: The GWAS study has identified a single locus of major effect contributing to beech bark disease resistance. Knowledge of this genetic locus contributing to resistance might be used in applied breeding, conservation and restoration programs.

Keywords: Beech bark disease, Association mapping, Resistance genes, American beech

\section{Background}

American beech (Fagus grandifolia Ehrl.) is native to the eastern North American deciduous forests and is the only species of this genus in North America [1]. The slow-growing, deciduous tree usually reaches about $37 \mathrm{~m}$ height $(120 \mathrm{ft})$ and may attain ages of 300 to 400 years [2]. The native range of American beech is within an area from Nova Scotia in southeastern Canada, west to Wisconsin and south to eastern Texas and northern Florida in the United States. Beech wood

\footnotetext{
*Correspondence: dbneale@ucdavis.edu

1 Department of Plant Sciences, University of California, Davis, CA 95616, USA

Full list of author information is available at the end of the article
}

is easily workable, excellent for turning and steam bending and used for flooring, furniture, veneer and containers [2].

Genetic research has been centered mostly on three genera (Fagus, Castanea and Quercus) of the family Fagaceae. The American beech (Fagus grandifolia Ehrh.) genome is estimated at $610 \mathrm{Mbp}$ [3] and has yet to be sequenced. The number of chromosomes is generally stable within the Fagaceae family $(2 \mathrm{n}=24)$, with occasional changes $(2 \mathrm{n}=24+1,2,3)$ resulting from irregular segregation at mitosis [4]. Both Fagus grandifolia and $F$. sylvatica have the most rudimentary genomes within the family, making their genomes attractive for comparative genomics studies [3]. 
The thinness of beech bark makes it vulnerable to a range of scale insects. Beech bark disease (BBD) is a scalefungal complex disease, initiated when a specific scale insect, Cryptococcus fagisuga Lind, attacks the bark of beech trees and renders it susceptible to bark canker fungi of the genus Neonectria [5]. Neonectria (Neonectria faginata or Neonectria ditissima) is the most common genus of ascomycete fungi associated with beech bark disease [6-8]. The disease develops through feeding activity of the beech scale insect that creates points of entryway for the fungal pathogen. Once established on the tree, the fungus is no longer influenced by fluctuations in beech scale density caused by environmental factors or habitat quality [9]. The cankering response of beech to fungal infection produces bark microstructure fissures and when it progresses the cankers may coalesce girdling or partially girdling the tree leading to wilting foliage and even mortality of trees. Individuals that survive infection are more susceptible to abiotic and biotic stress [10].

Beech bark disease first occurred when the scale insect was accidently introduced on plant material imported to Nova Scotia from Europe around 1890 [11]. It is found throughout northeastern U.S. states and southeastern Canadian provenances and is still expanding. The disease distribution is generally attributed to the initial phase of the insect life cycle ("crawlers") just after hatching, which is the only mobile phase of the life cycle. The "crawlers" can move to other areas on the same tree and can be dispersed by wind, birds, animals or humans. The mortality rate in beech has been significant in areas throughout the eastern United States. The loss of beech trees in some areas, where other hardwood species are rare, is causing even greater impact on wildlife, especially for black bear (Ursus americanus) [6, 12]. Pesticide control proved to not be fully effective in reducing the number of scale insects due to their protective waxy covering [13]. Neither pesticide application nor removal of infested trees is practical in large natural areas, because of labor, financial and environmental constraints [14]. Attempts to control $C$. faggisuga using a bio-agent such as a predatory mite [Allothrombium mitchelli Davis (Acari: Trombidiidae)] is currently under investigation [15].

Several field trials showed some beech trees remain after infestations that appear to be naturally resistant to BBD, usually clustered in small groups [16, 17]. About $1 \%$ of American beech trees remain disease free in forests long-affected by BBD [17]. There have been a small number of studies to understand the genetics of resistance of American beech to BBD [17-20]. Several studies have been conducted using molecular markers such as isozymes, RAPD (random amplified polymorphic DNA), AFLP (amplified fragment length polymorphism) and SSRs (simple sequence repeat) to differentiate between resistant and susceptible individuals and identify markers correlated with resistance, evaluate spatial and population genetic structure, and perform parentage analysis $[19,21-24]$. Although genetic marker studies enhanced the efforts to clarify modes of inheritance, no markers have been discovered that correlate with resistance. To estimate heritability, resistance to the beech scale insect, the artificial infestation technique developed by Houston [16], was used to test parent trees and their full and half-sibling progeny for resistance [24]. Individuals were classified as susceptible if five or more scale insects were present on the bark surface 1 year after scale insect eggs were affixed to the test tree. Low levels of resistance were found in families with only one resistant parent and a higher proportion of resistant progeny were only observed in families where both parent trees were resistant, confirming that resistance to beech scale insect is a heritable trait $[19,24]$. Current screening for resistance aims to increase the proportion of resistant trees and remove the susceptible trees for breeding purposes. These results formed the basis of a regional breeding program for BBD-resistant American beech [5, 25]. Identification of genetic markers associated with the resistance phenotype could potentially accelerate breeding efforts and reduce costs through the implementation of indirect selection methods, reducing the need for the costly, time consuming and labor intensive methods currently used to test trees for resistance to the scale insect.

Association mapping (AM) is an alternative approach that may, in theory, overcome limitations of pedigreebased quantitative trait loci (QTL) mapping [26]. It has been used in model species with available genomic resources, however with recently available genome sequences for forest species, this approach has the potential to increase the chance of understanding the genetic architecture of complex traits. The candidategene-based approach has been used for forest species where genome sequences were not yet developed [27]. In our study, we conducted a genome wide association study (GWAS) to discover loci associated with BBD resistance. A case-control design was used, which compared marker frequencies between a group of affected individuals (cases) and a group of unaffected individuals (controls). In this approach, we aimed to examine genetic architecture of disease resistance in American beech and identify candidate genes associated to BBD. Once identified, a marker breeding based approach could be used in breeding programs for BBD resistance.

\section{Methods}

\section{Transcriptome}

Plant materials

Trees sampled for RNA preparation were all part of the U.S. Forest Service Northern Research Station's American beech breeding program in Delaware, $\mathrm{OH}$. 
Tissues were sampled in the summer of 2009 from selected trees growing in naturally forested areas, grafted ramets of parent trees, and seedling progeny. The selected trees, or grafted ramets of the selected trees, were previously tested for beech scale resistance using an artificial infestation procedure [25]. To maximize genetic diversity, five resistant and five susceptible trees originating from a diverse geographic range, that included New Brunswick (Canada), Maine (USA), the lower peninsula of Michigan (USA), the upper peninsula of Michigan (USA), Pennsylvania (USA), and both northern and southern Ohio (USA) were selected for RNA sequencing and SNP discovery. Outer bark tissues including periderm, vascular cambium, and phloem, were harvested using grafting knives sterilized with liquid ethanol and were immediately frozen in a dry ice ethanol bath before transfer to a $-80{ }^{\circ} \mathrm{C}$ freezer until shipping overnight to the Schatz Center at Pennsylvania State University in a dry nitrogen shipper to prevent thawing.

\section{RNA preparation}

RNA samples were sequenced using Roche 454 sequencing technology. The main aim was to establish a database for network analysis to determine tissue specific expression patterns. Individual total RNA samples were prepared from bark tissues using the method first described by [28] and modified by [29]. At least five grams of frozen bark tissues were weighed, ground to a fine powder under liquid nitrogen, and dispersed in $\mathrm{CTAB}$ buffer. Following two chloroform extractions, RNA was precipitated with $\mathrm{LiCl}_{2}$, extracted again with chloroform and precipitated with ethanol. The resulting RNA pellet was re-suspended in 40-100 $\mu \mathrm{l}$ of DEPC-treated water, and the quality was assessed with an Agilent Technologies 2100 Bioanalyzer (Agilent Technologies). Poly(A) RNA was purified from total RNA using the Ovation RNA-Seq System kit (NuGen) following supplier's instructions. Reverse-transcription was performed using the Just cDNA kit (Stratagene) and random hexamer primers.

\section{Library construction and 454 Roche sequencing}

Individual sequencing libraries for each cDNA preparation were constructed and sequenced using a 454 sequencer as previously described [29]. The cDNA preparations were sheared to approximately $500 \mathrm{bp}$ fragment lengths. Adaptor sequences containing unique barcodes for each library were ligated to the fragmented cDNAs and immobilized on beads. The libraries for the five disease-resistant trees were pooled and the libraries for the five diseasesusceptible trees were combined for separate multiplex sequencing, each on a different half of the same plate. Multiplex sequencing of the library pools was performed on an FLX model 454 DNA sequencer (Roche Diagnostics) at Penn State University. The DNA sequence files for each of the ten cDNA libraries were selected and compiled from the batch sequencing raw data files using a Newbler 454 software utility, based on the unique barcodes assigned to each of the libraries. Sequences generated in this study were submitted to the Short Read Archive at the National Center for Biotechnology Information, accessions numbers SRX1781388 to SRX1781397, for NCBI BioProject Accession PRJNA321730 (NCBI: http://www.ncbi.nlm.nih.gov).

\section{Transcriptome assembly}

The SeqMan NGEN (DNASTAR) program for next generation transcriptome sequence data assembly was used to assemble contigs from the pooled 454 sequence data files for all five BBD-resistant tree libraries. Similarly, the pooled dataset consisting of 454 sequence data files for the five BBD-susceptible tree libraries was also assembled using the NGEN program. Finally, to obtain a reference transcriptome for $F$. grandifolia, all of the 454 sequencing data files for the ten libraries were pooled and assembled into a combined set of transcript contigs.

\section{Filtering of transcriptome assembly and SNP calling}

Mapping of the reads from each library onto the assembled transcriptomes revealed a high level of several sequences of ribosomal origins. These transcripts accounted for $34 \%(180,292$ out of 533,261$), 27 \%$ $(139,718$ out of 521,505$)$ and $18 \%(186,745$ out of $1,026,995)$ of the resistant, susceptible and combined assemblies, respectively. To determine the amount of structural non-coding RNA (ncRNA) sequences present in the libraries, the RFAM structural RNA database [30] was downloaded to serve as a local BLAST database. All of the sequence reads and contig sequences in the three assembled transcriptomes were aligned to the RFAM database, using a conservative BLAST $e$-value threshold of e-70, the ribosomal content was about $30 \%$ of the sequenced reads. ncRNAs accounted for 729, 1506 and 2110 contigs for the resistant, susceptible and combined assemblies, respectively. These contigs were removed from the assemblies, prior to SNP discovery.

Due to the large amount of ncRNA reads, another assembly was conducted using the Newbler program (Roche) which incorporated filtering for structural RNAs, low quality reads, and abbreviated reads. The BLAST cut-off $e$-value used in the filtering ncRNAs was set at e-50, resulting in 349,613 out of 1,406,316 reads in total to be removed prior to assembly. Again, assemblies were conducted for the 5 BBD-resistant libraries, the 5 BBD-susceptible libraries, and all 10 libraries combined.

Detection of putative polymorphic sites was performed by mapping reads filtered for structural RNAs to the 
beech reference transcript contigs, using the Newbler's gsMapper program. Both DNASTAR and Newbler alllibrary combined contig sets were used, separately, as reference transcriptomes in the SNP discovery. A minimum depth of coverage of 15 reads from each library was required to call putative SNPs. High confidence SNP sites were identified as those with a minimum of 100 bases flanking the SNP site and where the reference nucleotide at the SNP site was non-ambiguous. The stress-response genes and EST-based DNA markers served as a resource (Fagus grandifolia Transcriptome, Hardwood Genomics Project, www.hardwoodgenomics.org) for the construction of linkage maps and a framework for a GWAS study.

\section{Population sampling and phenotypic classification}

Resistant trees $(R=254)$ and susceptible trees $(\mathrm{S}=260)$ were located and positions mapped in six US states and nine stands. Trees in Penobscot county (Maine), Berkshire county (MA), Randolph county (WV) and Pascataquis county (ME) and two Canadian provenances: Prince Edward Isl. Canada, Kings county, and Sissiboo Falls, Digby county (NS) were located and mapped as reported in previous studies [18, 22]. Additional trees were located and mapped in Ludington State Park, Mason county (MI), Luce county (MI), Mckean county (PA), Clearfield county (PA), Clinton county (PA) and Licking county (OH) (Fig. 1).

Phenotypes were determined for 514 individuals through field assessment of all trees at the time of tissue collection. Trees exempt of beech scale insects and any apparent signs of fungal infection were classified as resistant. A subset of trees was artificially inoculated with scale eggs to confirm resistance either in the field or on grafted ramets of the original trees $[16,17,25]$. A complete description of sampled trees and their phenotypes is included in Additional file 1.

\section{Tests of association SNP genotyping}

Extraction of DNA from leaf and dormant bud tissues was carried out as described in [19]. Dormant buds were

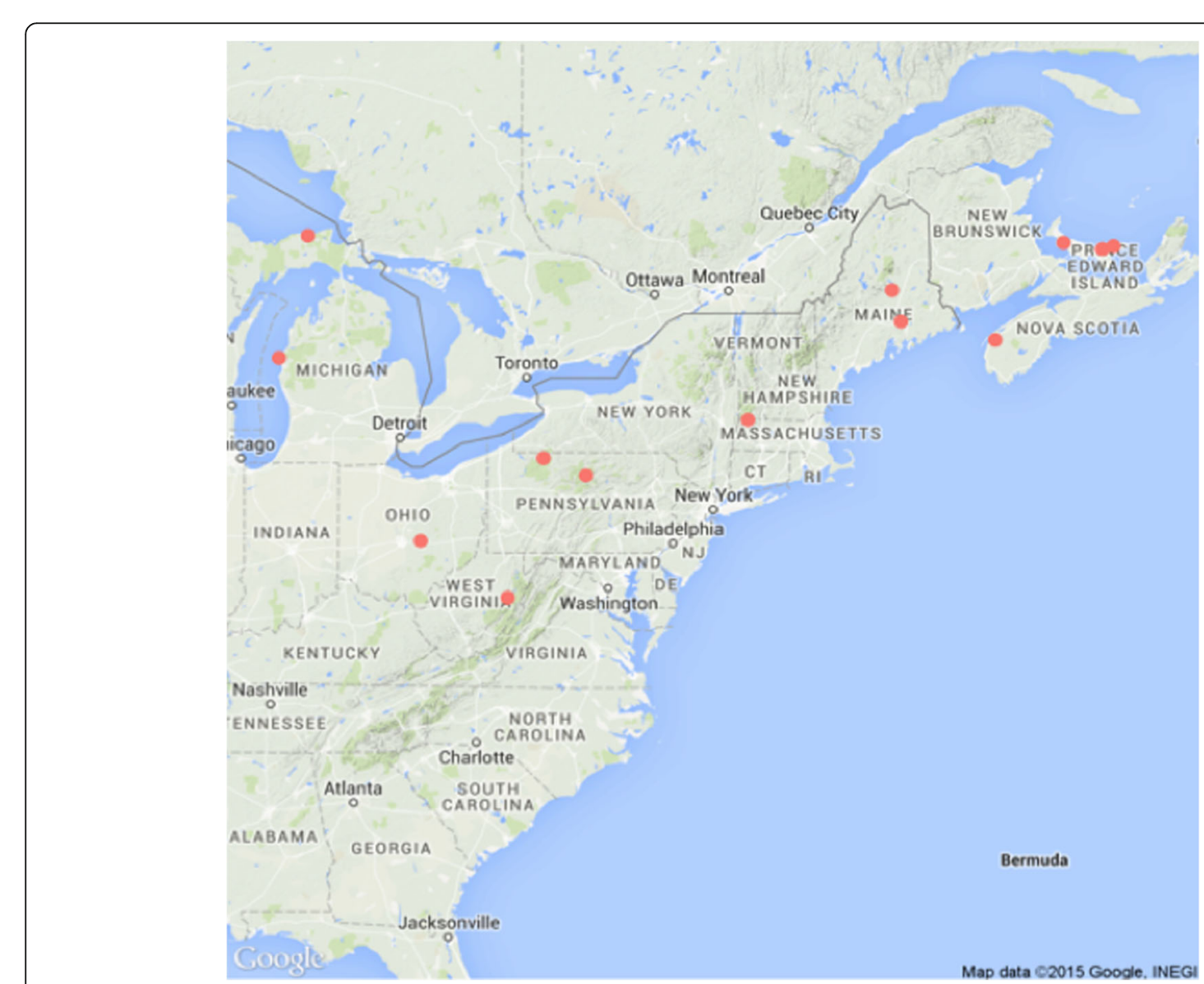

Fig. 1 Sampling locations of mapping population. Highlighted by red full circles are sampling locations of American beech full-sib individuals used for association mapping study across stands in six U.S. states and two Canadian provinces. The map graphic was reproduced in the package 'ggmap' (Spatial visualization in ggplot2) v.2.6.1 (Kahle and Wickham) [38] in software R with Google Maps and Stamen Maps. For Fig. 1 a copyright permission was not required 
collected as described in [18] and stored at $-70{ }^{\circ} \mathrm{C}$ until used for DNA extractions. For the 514 samples, DNA quality and quantity was tested before submitting for genotyping. Approximately $20 \mathrm{~K} \mathrm{SNPs,} \mathrm{originating} \mathrm{from}$ EST transcriptome assemblies, were submitted for design scores and $16 \mathrm{~K}$ of these were selected for inclusion on an Affymetrix Axiom ${ }^{\mathrm{mm}}$ Genome-Wide $1.5 \mathrm{~K}-50 \mathrm{~K}$ array (Santa Clara, CA). Genotypes were successfully determined for 506 of the 514 samples and were used in downstream analysis with an initial call rate of $97 \%$ or greater.

\section{SNP linkage map}

Controlled cross-pollinations were carried out between two confirmed beech scale resistant American beech trees $1505(\mathrm{R})$ and $1504(\mathrm{R})$ in Ludington State Park, MI (USA) as previously described to create the mapping population [31]. Additional cross-pollinations were carried out on grafted containerized ramets of the parents in 2010 to supplement the original family $(N=46)$, bringing the total number of progeny to 117 .

Linkage analysis was performed on 115 of the 117 progeny that were successfully genotyped at 5838 SNPs sites. A genetic map was constructed to order SNP markers used in association testing and served as graphical displays of the genome wide significant associated SNPs. The map construction was performed using the software Join-Map 2.0 [32]. All SNPs that successfully "passed" a 1:2:1 or 1:1 segregation ratio test in the offspring, were used in the linkage analysis, assigned to linkage groups and ordered to determine map genetic distances in $\mathrm{cM}$ (centimorgans).

\section{Population structure analyses and genome-wide identity- by-state test (IBS test)}

Discriminant Analysis of Principal Components (DAPC) was used to cluster individuals based on genotypes. This well-known method aims to maximize group differences while minimizing within cluster variances [33]. DAPC was applied on a matrix composed of 506 individuals and 5838 SNPs using an implementation available in the $\mathrm{R}$ package adegenet 2.0.0. To identify clusters, the principal component analysis (PCA) of the matrix data was first computed followed by Discriminant Analysis on the number of retained principal components as provided by DAPC method.

A test for population stratification was also performed with qqman package [34] in R software v.3.2.0, a common tool to visualize GWAS results and estimate the rate of genomic inflation. We measured genomic inflation also defined as $\lambda$ (lambda) to provide evidence of population stratification or cryptic relatedness.

To avoid statistical biases during population structure analysis and IBS score computations, SNPs with
Pearson's correlation coefficients $\left(\mathrm{r}^{2}\right)$ higher than 0.8 were rejected. Due to the absence of a genetic scaffold, a chromosomal location-based SNP filtering, as implemented in common GWAS packages, such as PLINK v.1.9 [35], was replaced by iterative SNP filtering. Pairwise Pearson's $r^{2}$ across SNPs were computed and, at each step during the iteration, the SNP correlated $\left(\mathrm{r}^{2}>0.8\right)$ with the most number of other SNPs was rejected. The iteration stopped when no remaining SNPs were correlated (all pairwise $\mathrm{r}^{2}<0.8$ ). This procedure left 3220 SNPs out of original 5838 SNPs. Filtered SNPs were then used to compute IBS scores, namely pairwise Pearson's correlation coefficients $\left(\mathrm{r}^{2}\right)$, between individuals. Here again iterative filtering was applied across trees to prune individuals with IBS $>0.1875$. Out of 506 trees, 327 passed IBS-based filtering.

\section{GWAS}

A logistic regression model was used to perform association tests between SNPs and disease scores. Population stratification was controlled using the first 20 ancestry principal components (PC's) as covariates in the logistic model. GWAS was performed using the PLINK 1.9 package ([35], https://www.cog-genomics.org/plink2, [36]) and 3220 SNPs on 327 independent individuals (172 cases and 155 controls, all pairwise IBS < 0.185). Prior to association testing, individuals with missing genotype rate $>10 \%$ were deleted, leaving effectively 172 cases (susceptible or diseased trees) and 155 controls (resistant or symptom-free trees). SNPs were then filtered for Hardy-Weinberg equilibrium ( $p$-values $>10^{-5}$ ), minor allele frequencies (MAF > 0.05) and missing genotypes across individuals $(<10 \%)$. In total, 3155 SNPs were included in the association test after filtering. The genotyping rate was equal to 0.99 . For every SNP included in the case-control test, the exact $P$ value $[\mathrm{P}]$ and the estimated odds ratio $[\mathrm{OD}]$ for the association between the minor allele [A1] and the disease phenotype were calculated. Resulting $p$-values underwent genomic inflation control.

\section{Linkage disequilibrium}

Haplotypes were identified using Haploview (v.4.2) [37], and with default parameters (exclusion of markers separated by $>500 \mathrm{~kb}$ and individuals with $>50 \%$ missing genotype). Pearson's coefficient of determination $\left(\mathrm{r}^{2}\right)$ was used to determine the pairwise correlation between genetic markers.

\section{Results}

\section{Transcriptome results}

RNA sequence data

The sequencing of $10 \mathrm{cDNA}$ libraries yielded 1,406,316 reads covering 508,764,432 bases. The libraries from BBD-resistant trees yielded between 87,964 and 210,340 
reads, while 70,218 to 205,945 reads were obtained for libraries from BBD-susceptible trees (Table 1). While the quality and quantity of the sequence data was acceptable (Table 1; Additional file 2), the mRNA poly-A selection step left a large proportion of structural non-coding RNAs in the samples, including ribosomal RNA. Moreover, up to a third of the transcriptome sequence reads were mapped to ncRNAs. Subsequently, the quality of the mRNA preparations was re-assessed with an Agilent Technologies 2100 Bioanalyzer (Agilent Technologies), revealing remnant rRNA peaks (Additional file 3 ). Because the ncRNA contamination was detected after the first transcript assemblies were conducted, a second assembly with additional filtering was conducted (see below).

\section{Assembly of transcript contigs}

Assemblies of 454 sequence reads into contigs were conducted using both the SeqMan NGEN (DNASTAR) program and the 454 Newbler (Roche) assembler. Contigs were built using reads from, either five BB-resistant libraries, five BBD-susceptible libraries or combination of both libraries.

The NGEN assembly resulted in a total of 28,592, 27,544 and 44,065 contigs for pools of BBD-resistant, BBD-susceptible and the combination of both libraries, respectively. NGEN assemblies incorporated 76\%, 73\% and $73 \%$ of the sequence reads from the BBD-resistant, BBD-susceptible and the combined samples. NGEN contig lengths averaged approximately 360 bases, with a median varying from 475 to 542 bases. The longest transcripts obtained in the resistant, susceptible and combined assemblies were 11,704, 8168 and 10,800 bases, respectively. Table 2 summarizes the results of the three $F$. grandifolia transcriptome assemblies using the NGEN program.

The Newbler filtered assembly resulted in 10,690 contigs for the BBD-resistant data, 7630 contigs for the BBD-susceptible data, and 16,285 contigs for all libraries combined. The Newbler assemblies incorporated 84\%, $81 \%$ and $86 \%$ of the sequence reads from the BBDresistant, BBD-susceptible and the combined data, respectively. The longest transcripts obtained by Newbler in the resistant, susceptible and combined assemblies were 4651, 4336 and 10,681 bases respectively. The average length of the Newbler contigs across the three assemblies was 679 bases. The detailed Newbler assembly statistics are shown in Table 3. The "large contigs" from the Newbler assembly are those with 500 bases or longer, which overall averaged 949 bases in length.

\section{Single nucleotide polymorphism (SNP) discovery}

SNP site discovery was performed using both the DNASTAR NGEN and Newbler combined assemblies as reference transcriptomes. The Newbler gsMapper SNP calling program generated two output files. One output file contained all possible SNPs. The other output file contained only the high confidence SNP calls, which was used as the starting point for selecting SNPs for the mapping study. A summary of the SNP discovery results is displayed in Table 4. As shown in the last two columns of Table 4, 15,542 and 12,119 candidate SNP sites were discovered using the Newbler and DNASTAR reference transcriptomes, respectively. For each candidate SNP with a minimum 100 bases of sequence flanking the SNP, 50 bases from each flanking side of the SNP site was extracted for DNA marker development. The

Table 1 Result metrics for the sequencing of 10 beech cDNA libraries

\begin{tabular}{llll}
\hline Library Name (based on tree number) & Number of reads & Average read length & Total bases \\
\hline BBD-Resistant trees & & & 375 \\
Beech_1228R & 210,340 & 354 & $78,912,594$ \\
Beech_2692R & 131,306 & 370 & $46,467,779$ \\
Beech_1504R & 123,194 & 358 & $45,498,709$ \\
Beech_1208R & 87,964 & 374 & $31,467,605$ \\
Beech_2276R & 147,781 & 366.2 & $55,192,118$ \\
Resistant tree Sub-Totals & 700,585 & & $257,538,805$ \\
BBD-Susceptible trees & & 358 & $73,702,265$ \\
Beech_1973S & 205,945 & 349 & $55,759,976$ \\
Beech_DN00726S & 159,660 & 359 & $46,933,567$ \\
Beech_3128S & 130,917 & 357 & $49,586,742$ \\
Beech_2143S & 138,991 & 360 & $25,243,077$ \\
Beech_Holden & 70,218 & 356.6 & $251,225,627$ \\
Susceptible tree Sub-Totals & 705,731 & 361 & $508,764,432$ \\
Totals for all libraries & $1,406,316$ & & \\
\hline
\end{tabular}


Table 2 F. grandifolia transcriptome NGEN assembly statistics

\begin{tabular}{llll}
\hline & BBD-Resistance Libraries & BBD-Susceptible Libraries & "Combined" Reference transcriptome \\
\hline Assembled Reads & 533,261 & 521,505 & $1,026,995$ \\
Unassembled Reads & 167,324 & 184,226 & 379,321 \\
Total Number of Reads & 700,585 & 705,731 & $1,406,316$ \\
Assembled Reads (\%) & 76.12 & 73.90 & 73.03 \\
Assembled Contigs & 28,592 & 27,544 & 44,065 \\
Contigs $>2$ K & 622 & 271 & 1115 \\
Av. Length of Contigs & 362 & 354 & 357 \\
\hline
\end{tabular}

values in parenthesis in the last two rows of Table 4 represent the number of SNP sites selected, based on sufficient flanking sequence. The sequences and statistics for the high quality and most informative SNPs used in the GWAS are presented in a table in Additional file 4.

\section{Test of association}

\section{SNP genotyping data results}

In total, 514 DNA samples were submitted to genotyping using the Affymetrix Axiom ${ }^{\text {rm }}$ Genome-Wide $1.5 \mathrm{~K}$ $50 \mathrm{~K}$ array and after quality filtering, resulted in genotypes for 506 samples $(R=249$ and $\mathrm{S}=257)$. Of the initial 16,709 SNPs, 5838 Poly High Resolution SNPs passed Affymetrix filtering metrics and only these were included in downstream analysis. A set of 5838 SNPs was visualized for the cluster pattern in SNPolisher $R$ Package v.1.5.1 (Affymetrix Inc.).

\section{SNP linkage map construction}

We developed a single-nucleotide polymorphism (SNP) - based linkage genetic map for American beech. Single locus Mendelian segregation was first tested using $X^{2}$ goodness-of-fit to 1:2:1 and 1:1 ratio at $5 \%$ and $1 \%$ significance levels. Linkage analysis produced 12 linkage groups (Fig. 2) using JoinMap 2.0 [32]. Out of 3236 SNPs apparently segregating, 16
SNPs failed to be linked so the final number of linked SNPs was 3220 (Additional file 5).

\section{GWAS}

Population structure analysis and IBS test results

To explore the population structure of our sample population, Discriminant Analysis of Principal Components (DAPC) was applied to 506 individuals and 5838 SNPs. DAPC revealed three genetic clusters (Additional file 6A) using 40 principal components (PCs), maximum numbers of clusters and discovery clusters limited to 40 and 7, respectively and 6 discriminants. In addition, we employed a genomic control to assure for population structure by estimating an inflation factor $\lambda$ (genomic control measures). Significant inflation was detected based on a QQ-plot of association $p$-values, which displayed systematic deviation from the expectation (Additional file 6B).

The pairwise clustering based on identity-by-state (IBS), revealed high correlation among individuals in the sample population. The IBS test allowed the removal of individuals with the highest number of correlated "partners", indicating high likelihood of relatedness. Our method of choice was to test for population outliers by performing IBS-based nearest neighbor analysis. In total, 179 individuals from the

Table 3 F. grandifolia transcriptome sequence assembly summary obtained from Newbler

\begin{tabular}{llll}
\hline Assembled Contig Sequences & Beech "Resistant" Library & Beech "Non-Resistant" Library & Beech "Combined" Library \\
\hline Aligned Bases (\%) & $157,365,926$ & $143,162,558$ & $314,816,063$ \\
& $(83.63 \%)$ & $(80.91 \%)$ & $(86.23 \%)$ \\
Aligned Reads (\%) & 438,153 & 412,164 & 893,216 \\
& $(82.04 \%)$ & $(78.90 \%)$ & $164.54 \%)$ \\
Number of Contigs, All & 10,690 & 7630 & $11,664,012$ \\
Total Contig Bases & $7,845,700$ & $4,478,078$ & 716 \\
Average Contig Length & 734 & 587 & 9943 \\
Number of Large Contigs & 7147 & 4018 & 1009 \\
Average Large Contig Size & 961 & 875 & 1081 \\
N50 Large Contig Size & 1005 & 893 & 10,681 \\
Largest Contig Size & 4651 & 4336 & \\
\hline
\end{tabular}


Table 4 Summary of SNP discovery results for F. grandifolia using the reference transcriptomes generated by DNASTAR NGEN and Newbler

\begin{tabular}{lll}
\hline Statistics & DNASTAR Reference Transcriptome & Newbler Reference Transcriptome \\
\hline Number of Contigs in Reference & 43,212 & 14,977 \\
Number of Bases in Reference & $28,676,242$ & $11,580,835$ \\
Number of Mapped Reads (\%) & $1,357,629(96.55 \%)$ & $938,418(88.82 \%)$ \\
Number of Mapped Bases (\%) & $494,805,288(97.45 \%)$ & $312,081,222(85.48 \%)$ \\
Fully Mapped Reads (\%) & $444,838(31.64 \%)$ & $396,609(37.54 \%)$ \\
Partially Mapped Reads (\%) & $35,728(2.54 \%)$ & $135,858(12.86 \%)$ \\
Non-Unique Mapped Reads (\%) & $871,685(61.99 \%)$ & $147,210(13.93 \%)$ \\
Chimeric Reads (\%) & $5378(0.38 \%)$ & $258,741(24.49 \%)$ \\
Unmapped Reads (\%) & $32,025(2.28 \%)$ & $101,623(9.62 \%)$ \\
Reads Too Short (\%) & $16,470(1.17 \%)$ & $16,470(1.56 \%)$ \\
High Confidence SNP Calls & $2119(12,069)$ & $15,542(14,574)$ \\
HC SNP Calls (Not Ambiguous) & $10,971(10,934)$ & $15,541(14,573)$ \\
\hline
\end{tabular}

five different stands were identified as possible very close relatives and were removed from the further downstream analysis (Table 5).

\section{Logistic regression test}

The initial logistic regression test was performed with 3220 SNPs, however after filtering SNPs to compute PCA and IBS score, an independent set of 2116 SNPs remained. No Genomic inflation from GWAS $p$ values expected by random chances was detected, except for the top associated SNPs (lambda $=1.13$, Fig. 3a). A Fisher's exact test revealed four markers on chromosome 5 (Fig. 3c), whose $P$ values were above the significant genome-wide threshold of ( $P$ value $>1.585 \times 10^{-5}$ ) (Fig. 3b; Additional file 7). For the association test, the significance threshold for all 3155 SNPs was Bonferroni's $\left(\alpha^{*}=\alpha / n\right)$ based significant threshold to adjust for multiple testing, where $\alpha$ represents Bonferroni's coefficient 0.05 and $n$ represents the number of SNPs after filtering for quality parameters $\left(0.05 / 3155=1.58 \times 10^{-5}\right)$.

\section{Identification and mapping of the disease resistance candidate gene}

As shown in the Manhattan plot (Fig. 3b and Additional file 7), four SNPs were observed to surpass the genome-wide significance threshold of $1.585 \times 10^{-5}$, which is strong evidence of association. All four SNPs are located on chromosome (Chr) 5 (Additional file 8). The strongest evidence of association is linked to AX-156994126 $(P=5.99 \mathrm{E}-6$, odds ratio $(\mathrm{OR})=0.2573), \mathrm{AX}-156988334(P=8.852 \mathrm{E}-6$, odds ratio $(\mathrm{OR})=0.2758)$ and $\mathrm{AX}-157000652$ $(P=8.074$ E-6, odds ratio $(\mathrm{OR})=0.2773)$ (Table 6$)$. On chromosome (Chr) 5, SNPs were positioned at 12.344 cM (centimorgans) for AX-156994126, AX-
156989406 and for AX-157000652 and at $13.811 \mathrm{cM}$ for AX-156988334 (Additional file 8A).

The flanking sequences for these four SNPs were used in Blast analysis. The best BLAST (BLASTn) analyses were performed against the NCBI database (National Center for Biotechnology Information) for non-redundant protein database. The best hit resulted in the identification of the single gene $(M t)$ from a single contig (contig 03321), within which fell all four identified SNPs (see Additional file 8B). The gene $(M t)$ encodes an mRNA for metallothionein-like protein (metal ion binding) (Table 6).

Support for the result from GWAS was obtained by BLASTx alignment of the RNA sequence reads for each of the 10cDNA libraries individually to contig 03321, containing the full length transcript of the candidate $M t$. gene. With the exception of the individuals (1504R and DN00726S), the constitutive expression of the candidate $M t$. gene was higher in the $\mathrm{BBD}$ resistant individuals than in the BBD susceptible individuals (Additional file 9). On average, 1602 reads mapped per BBD resistant library, and 414 reads per susceptible library, after normalization in TPM (Transcripts Per Kilobase Million). This does not imply the expression of the candidate $M t$. gene alone is sufficient for $\mathrm{BBD}$ resistance, nor that it is the only gene differentially expressed upon attack by the insect vector.

\section{Linkage disequilibrium}

To measure the degree to which alleles at two loci are associated, a complete set of 3220 SNPs were included to determine whether two loci are in linkage equilibrium or disequilibrium. LD plot showed SNPs in strong linkage disequilibrium ( $\mathrm{D}^{\prime}=1$ ) (Additional file 10). 


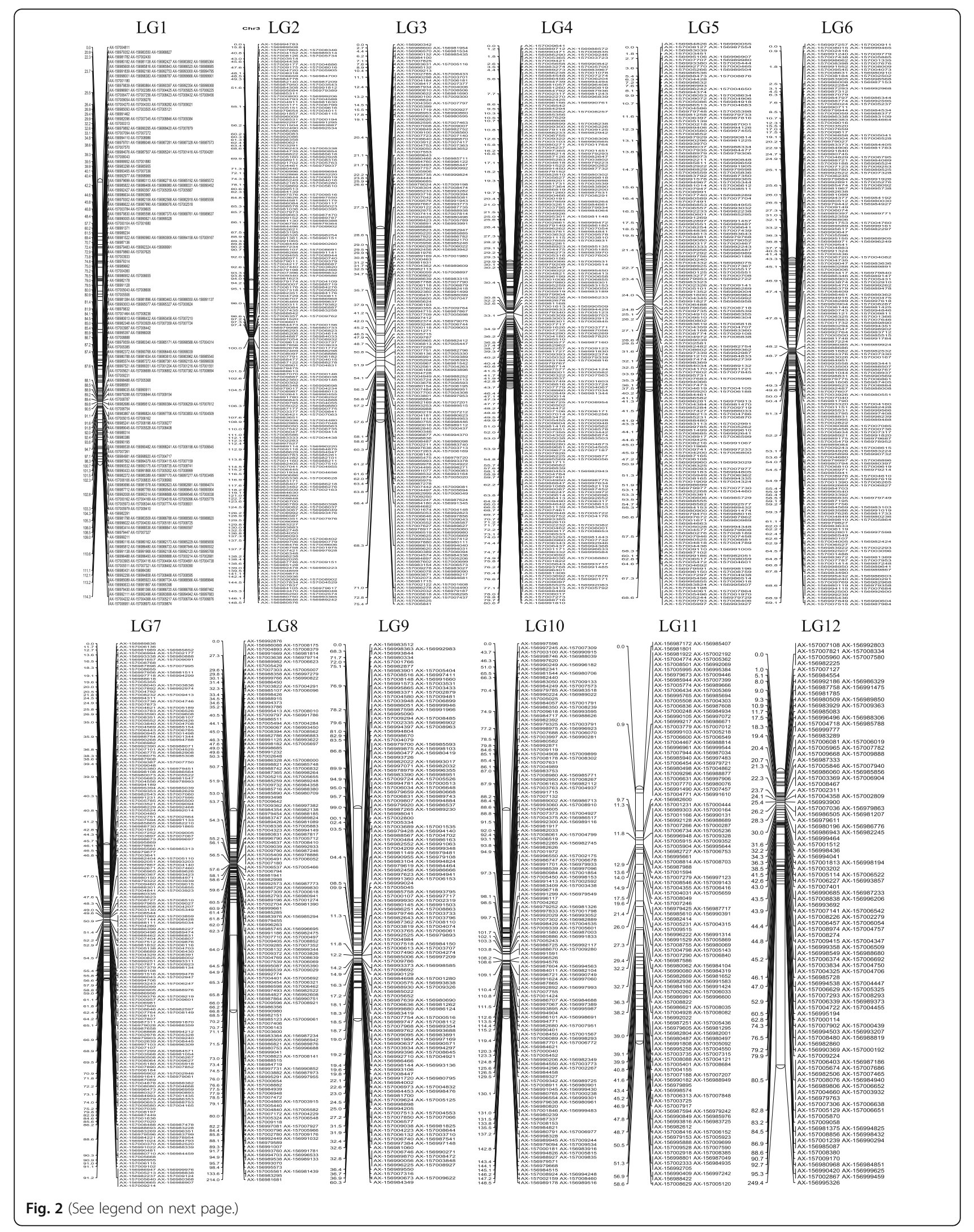


(See figure on previous page.)

Fig. 2 Genetic linkage map of F. grandifolia. Genetic linkage map of F. grandifolia constructed using 115 progeny individuals derived from the cross controlled experiment 1505 (R) $\times 1504$ (R). Totally 3220 single nucleotide polymorphism markers are linked in twelve groups and presented on the right side of each linkage group. Map distances in centi-morgans are presented on the left side

\section{Discussion}

\section{Trait architecture}

Genome Wide Association Analysis has identified a single locus contributing to resistance to beech bark disease (BBD). There were four SNPs in chromosome (Chr) 5 significantly associated with the scale resistance trait analyzed. A candidate gene $(M t)$ encoding for a metallothionein-like protein was found to be physically linked to these genetic markers and may play an important role in the resistance mechanisms against Nectria sp. - beech scale insect. This is consistent with genetic studies of several different small full-sibling families that suggest involvement of a few as two genes [5, 24, 38]. For validation of single locus trait discovery, BLASTn search of the contig EST sequences was performed against the complete NCBI database for those SNPs (see Additional file 8B). A proven functional annotation for these SNPs is essential prior to use in breeding, which will be possible when a reference genome sequence for American Beech is available.

Disease resistance in plants can involve any number of genes, from a single major gene to many loci determining resistance. Single-gene resistance mechanisms with large effects are more common in agricultural crops but only a few have been described in forest species, which reflects the greater genetic diversity of the host and pathogen populations in forest pathosystems [39]. In forest species, resistance is typically polygenic and durable, with few examples of simply inherited disease resistance. This is likely due to the limited potential for Mendelian analysis in forest trees and complex life cycles of many forest pathogens [40]. However, disease resistance is not exclusively polygenic in forest pathosystems. Examples of single qualitative resistance, include loblolly pine (Pinus taeda) resistance to the fusiform rust disease $[41,42]$, resistance to white pine blister rust in several species of pine $[43,44]$ and evidence for major gene resistance to weevil in Sitka spruce [45].
In this study, we used Affymetrix Axiom ${ }^{\mathrm{Tm}}$ GenomeWide $1.5 \mathrm{~K}-50 \mathrm{~K}$ array (Santa Clara, CA) to genotype 327 individuals used for association mapping. Although SNP discovery was performed specifically for BBD, a very small proportion of the SNPs deemed informative in downstream analysis. The conversion rate, provided by Affymetrix genotypng facility, corresponded to $34.04 \%$ and was quite high compared to Pinus taeda (Loblolly pine) at only $5-10 \%$. Overall, the number of informative SNPs was sufficiently high to provide us with association power on the genome scale for the disease resistance.

The knowledge of genetic architecture is important for breeding resistant varieties to develop resistant planting stock for restoration of impacted habitats. Molecular markers have also contributed to improved breeding strategies for monogenic resistance genes when combining them in a "gene pyramiding" strategy for a more durable resistance [46] and can also be used to develop cost-effective indirect selection techniques.

\section{Candidate gene role}

Plant metallothioneins are proteins thought to sequester excess amounts of certain metal ions [47]. These low molecular weight proteins $(4-8 \mathrm{kDa})$ were discovered in mature wheat embryos about 30 years ago [48]. Metallothioneins represent Cys-rich metal chelators able to coordinate metals atoms (e.g. $\mathrm{Zn}, \mathrm{Cd}$ and $\mathrm{Cu}$ ions) and found to play a role in cellular processes such as regulation of cell growth, proliferation and DNA damage repair. But how metallothioneins fulfill these cellular roles, is yet to be discovered [49, 50]. Expression of plant metallothionein genes has been observed in a variety of senescing tissues, such as leaves and stems, ripened fruits and wounded tissues [49]. Recent reports show that MTs (metallothionein's) are also involved in the scavenging of reactive oxygen species (ROS) [51].

Table 5 Duplicated individuals revealed by IBS test for the threshold (IBS > 0.1875)

\begin{tabular}{|c|c|c|c|}
\hline Stand & Number of excluded individuals & Disease status & \\
\hline Ludington State, Ml & 1 & R & NA \\
\hline Berkshire county, MA & 18 & $9 R$ & 95 \\
\hline Penobscot county, Maine & 55 & $33 R$ & $22 S$ \\
\hline Randolph county, WV & 38 & $26 \mathrm{R}$ & 125 \\
\hline Sissiboo Falls, Digby county, NS-Canada & 65 & $27 \mathrm{R}$ & 385 \\
\hline Not classified & 2 & NA & 25 \\
\hline Total & 179 & & \\
\hline
\end{tabular}



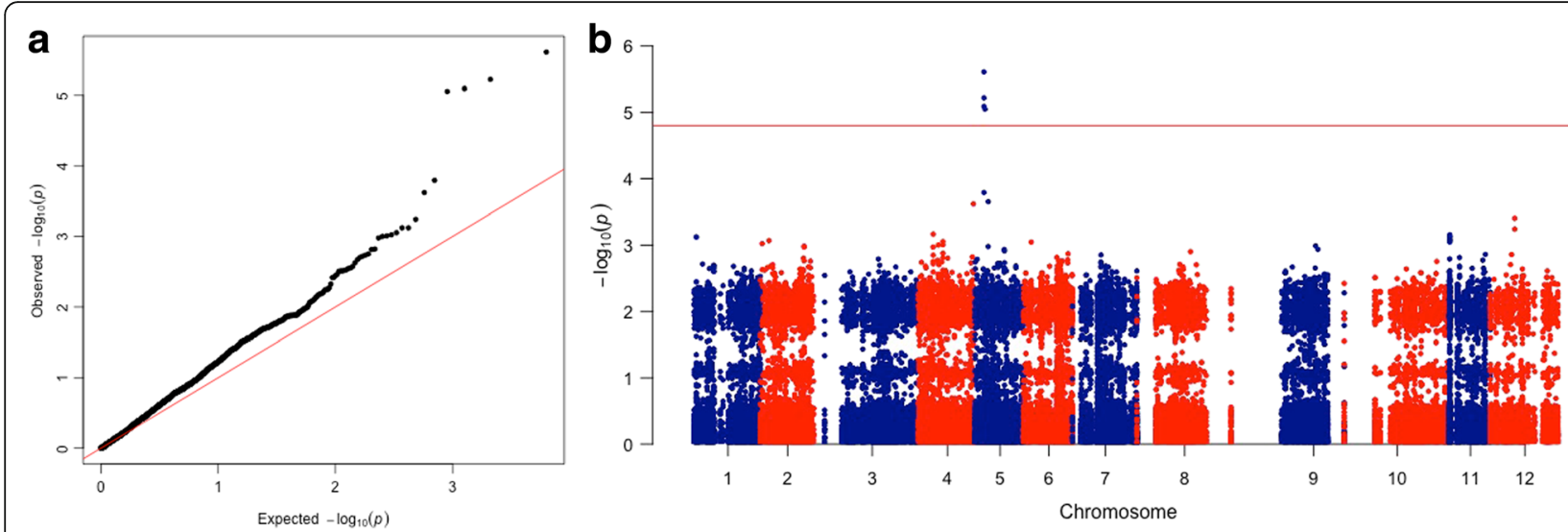

Fig. 3 a Quantile-quantile (QQ) plot of GWA p-values. QQ-plot shows only minor deviations from the null distribution, expected for the top associated SNPs. b Manhattan plot from the GWAS analysis of Beech Bark Disease in 327 individuals. Beech bark disease is associated with a locus on chromosome 5. The $x$-axis represents chromosomal locations and the $y$-axis, $-\log 10 p$-values from genotypic associations. Four markers on chromosome 5 reached genome-wide significance $\left(p\right.$-values $\left.>1.585 \times 10^{-5}\right)$

Metallothionein-like protein class II (Fagus sylvatica type) was described in Norway spruce (Picea abies), whose expression pattern was analyzed via ESTs from cDNA libraries [52]. Type 4 metallothionein-like protein genes are expressed in inner bark tissue of Japanese cedar (Cryptomeria japonica) [53]. ESTs encoding metallothionein-like proteins were the most frequently found hits in both early and late flushing libraries. Metallothionein-like protein activity is probably initiated by some cellular events during late flushing [52].

Changes in expression of metallothioneins and metallothionein-like proteins have been previously reported in response to biotic stresses in plants, including insect herbivory and fungal infections (reviewed in [53]). There is not consensus of the role of metallothioneinlike proteins in biotic stress response, but a role in oxidative stress have been proposed [53].

\section{Implementation of the findings in the future breeding program in beech}

A number of insects and diseases cause significant loss to forest productivity. Most of the current operational strategies for insect and disease control rely on classical breeding methods to develop populations enriched for resistance [54]. With emergence of genomics-based approaches, such as genome-wide association studies (GWAS) and genomic selection (GS), a broader range of applications is now available for plant breeding and genetic research $[55,56]$. In Fagus, mapping populations have been developed to discover QTLs for traits correlated to BBD. The tree improvement program included crosses to study inheritance of resistance to Cryptococcus fagisuga (see $[19$, 57]). However, association mapping like GWAS for QTLs underlying disease resistance to the BBD, has not been previously reported. In the present study, we used a GWAS mapping approach and a SNP linkage map to identify candidate resistance genes. To confirm the SNPs identified are truly associated with the scale-resistant trait, replication of this GWAS study is necessary, using independent casecontrol data from the initial population of unrelated individuals (see $[58,59]$ ).

Deployment of resistant planting stock can help to reduce disease incidence throughout natural stands of American beech. Markers found in this study that exhibit a significant association with the resistant phenotype, can be further refined to develop efficient and cost effective indirect selection techniques such as MAS (marker assisted selection) and genomic selection (GS) or combination of both (see [60]).

Table 6 Top SNPs associated with Beech Bark disease

\begin{tabular}{llllll}
\hline Gene & Chr & Position $(\mathrm{cM})$ & Affymetrix ID & Original SNPs ID & $\begin{array}{l}\text { Logistic regression } \\
\text { association }(P \text { value) }\end{array}$ \\
\hline Mt & 5 & 12.344 & AX-156989406 & contig03321_576 & 2.46E-6 \\
Mt & 5 & 12.344 & AX-157000652 & contig03321_166 & $8.07 E-6 \quad$ Fagus sylvatica (European beech) \\
Mt & 5 & 13.811 & AX-156988334 & contig03321_330 & $8.85 E-6$ \\
Mt & 5 & 12.344 & AX-156994126 & contig03321_441 & $5.99 E-6$ \\
\hline
\end{tabular}




\section{Conclusion}

To our knowledge, this is the first study designed to determine the genetic factors of disease resistance to beech bark disease (BBD) with genome scan analysis in American beech tree. The results presented identified four highly significant markers associated with a single locus located on chromosome (Chr) 5. All four loci were localized to the same contig within a single gene $(M t)$, that encodes for Fagus sylvatica mRNA for metallothionein-like protein (metal ion binding). Once a reference genome sequence is available, it will be possible to gain more insight into functional annotation of the four SNPs and determine the exact number of genes associated to BBD.

\section{Additional files}

Additional file 1: The list of sampled trees and their phenotypes. (XLSX $146 \mathrm{~kb}$ )

Additional file 2: RNA-seq data quality assessment. (PDF 853 kb)

Additional file 3: Examples of RNA vs. mRNA Agilent Bioanalyzer quality profiles. (PDF $288 \mathrm{~kb}$ )

Additional file 4: The sequences and statistics for the high quality and most informative SNPs used in the GWAS. (XLSX $1080 \mathrm{~kb}$ )

Additional file 5: The list of mapped SNPs in 12 linkage groups (LGS). (XLSX $91 \mathrm{~kb}$ )

Additional file 6: (A) DAPC analysis revealed three main genetic clusters where the individuals shown as dots and the groups as inertia ellipses. Eigenvalues of the analyses are displayed inset. (B) Quantile-quantile (QQ) plot of GWA $p$-values where on $x$-axis, are expected $-\log 10 P$ values and on $y$-axis observed $-\log 10 P$ values. The plot is showing large deviation from the null distribution where the inflation factor was higher than the threshold of 1, indicating a high genomic inflation in Beech association data and an existence of the population stratification. (PDF 90 kb)

Additional file 7: The statistics for Fisher exact test and Logistic regression test. (XLSX $4534 \mathrm{~kb}$ )

Additional file 8: (A) Four highlighted markers with significance level higher than genome-wide threshold $\left(P\right.$ value $\left.>1.585 \times 10^{-5}\right)$ located on the chromosome (Chr) 5. (B) Alignment of four nucleotide sequences to reference sequence Fagus sylvatica mRNA (Sequence ID: AJ130886.1). The FASTA sequence order corresponds as AX-156994126 (SEQ_1), AX156989406 (SEQ_2), AX-156988334 (SEQ_3) and AX-157000652 (SEQ_4). Highlighted red nucleotides refer to polymorphism to reference sequence and green nucleotides present diagnostics SNPs, respectively. (DOCX $4155 \mathrm{~kb}$ )

Additional file 9: RNA sequence reads from each cDNA library mapped to the full-length copy of the candidate gene transcript sequence from contig 03321, representing the expression of the candidate $M t$ gene after the challenge by the insect vector. (DOCX $161 \mathrm{~kb}$ )

Additional file 10: Linkage disequilibrium (LD) structure across four SNPs associated to BBD. (Red) strong LD between markers; (white) no LD. The block-like pattern represents the regions of high LD. Pairwise LD among four SNPs listed as squared allelic correlation (r2) [61] and Lewontin's D' [62]. (DOCX $162 \mathrm{~kb})$

\section{Abbreviations}

AFLP: Amplified fragment length polymorphism; AM: Association mapping; ARE: Antioxidant; BBD: Beech bark disease; BLAST: Basic alignment search tool; BLASTn: Nucleotide basic alignment search tool; cDNA: Complimentary DNA; Chr: Chromosome; CM: Centimorgans; CTAB: Cetyltrimethyl ammonium bromide; DAPC: Discriminant analysis of principal components; DEPC: Diethyl dicarbonate or diethyl purocarbonate; ERE: Ethylene; EST: Expressed sequence tag; GARE: Gibberellic acid; GS: Genomic selection; GWAS: Genome-wide association study; IBS: Identity-by-state; LD: Linkage disequilibrium; $\mathrm{LiCl}_{2}$ : Lithium chloride; MAF: Minor allele frequencies; MAS: Marker assisted selection; MeJARE: Methyl jasmonate; Mt:: Metallothionein-like protein (metal ion binding); NCBI: National Center for Biotechnology Information; ncRNA: Non-coding RNA; OD: Odds ratio; PCA: Principal component analysis; PCs: Principal components; Poly(A)RNA: Polyadenylation RNA; Q-Q plot: Quantile-quantile plot; QTL: Quantitative trait loci; RAPD: Random amplified polymorphic DNA; rRNA: Ribosomal nucleic acid; SARE: Salicylic acid; SSRs: Simple sequence repeat; TPM: Transcripts Per Kilobase Million; $\lambda$ : Lambda

\section{Acknowledgments}

Authors give a special thanks to Randi Famula for laboratory assistance on DNA preparation for genotyping, Mirko Ledda for comprehensive GWAS scripting and Christopher Campbell for assistance on computational scripting at UC Davis. Authors thank Tyler Wagner and Lynn Tomsho in the lab of SC Schuster at Penn State University for RNA preparations, cDNA library constructions and RNAseq data production. Authors thank to David Houston and Dan Houston for providing us the tissue of their earlier collected material of the isozyme research project. Authors acknowledge John E. Bowers for useful suggestions while reading the manuscript.

\section{Funding}

This work was supported by a grant from USDA Forest Service, Ohio (USDA FHP STDP grant - ID: NA-2009-01). The role of the funding body was to provide the design of the study and collection of data. The analysis, interpretation of data and writing of the manuscript was supported by Plant Science Department at UC Davis.

\section{Availability of data and materials}

All data generated or analyzed during the current study are included in this published article and its supplementary information files.

\section{Authors' contributions}

IC, JK and DN conceived the study and designed the experiments. JK and DC were involved in the development of the association mapping population. JK and DC performed phenotypic analysis. CA-Q and JC were involved in transcriptome assembly and development of EST transcriptome for American beech. Data analysis were performed by IC. The manuscript was prepared by IC, JK and DN. All the authors read and approved the final manuscript.

\section{Ethics approval and consent to participate}

Dormant bud tissue from beech trees in Penobscot County, ME; Berkshire County, MA; Prince Edward Island, Canada; Nova Scotia, Canada; and Randolph County, WV were previously collected by and were kind gifts from David Houston (Ohio State University, retired) and Dan Houston (U.S. Forest Service, Northern Research Station, retired). Additional details on these collections can be found in Houston and Houston [18]. Tissue samples from beech trees in Licking County, $\mathrm{OH}$ were collected with the permission of the Dawes Arboretum. Tissue samples from beech trees in Clearfield and Clinton Counties, PA were collected with permission of the Pennsylvania Department of Conservation and Natural Resources. Tissue samples from beech trees in McKean County, PA were collected with permission from the Allegheny National Forest. Tissue samples from beech trees in Luce and Mason counties, MI were collected with permission from the Ludington State Park. Tissue samples from beech trees in Pascataquis County, ME was collected with permission from the Maine Forest Service.

\section{Consent for publication}

Not applicable.

\section{Competing interests}

The authors declare they have no competing interests.

\section{Publisher's Note}

Springer Nature remains neutral with regard to jurisdictional claims in published maps and institutional affiliations. 


\section{Author details}

'Department of Plant Sciences, University of California, Davis, CA 95616, USA. ${ }^{2}$ USDA Forest Service, Northern Research Station, Forestry Sciences Laboratory, Delaware, $\mathrm{OH} 43015$, USA. ${ }^{3}$ Department of Horticulture and Landscape Architecture, Purdue University, West Lafayette, Indiana 47907, USA. ${ }^{4}$ Present address: Division of Natural Sciences and Mathematics, Lewis-Clark State College, 500 8th Avenue, Lewiston, ID 83501, USA. ${ }^{5}$ Schatz Center for Tree Molecular Genetics, Department of Ecosystem Science and Management, Pennsylvania State University, University Park, PA 16802, USA.

Received: 9 January 2017 Accepted: 7 July 2017

Published online: 20 July 2017

\section{References}

1. Fowells HA. American beech (Fagus grandifolia Ehrh.). In Silvics of Forest Trees of the United States, Agriculture Handbook No.271. Washington DC, US: Dept. Agric. Forest Service; 1965. p. 172-180.

2. Tubbs $\mathrm{CH}$, Houston DR. Fagus grandifolia Ehrh. American beech, Fagaceae beech family. In: Burns RM, Honkala BH, editors. Silvics of North America, vol. 2 hardwoods. Agriculture handbook 654, Vol. 2. Washington, DC: U.S. Department of Agriculture, Forest Service; 1990. p. 325.

3. Kremer A, Casasoli M, Barreneche T, Bodénès $C$, Sisco P. 5 Fagaceae trees. In: Kole $C$, editor. Genome mapping and molecular breeding in plants, volume 7, Forest trees. Berlin Heidelberg: Springer-Verlag; 2007. p. 162-87.

4. Zoldos V, Papes D, Brown SC, Panaud O, Siljak-Yakovlev S. Genome size and base composition of seven Quercus species: inter- and intra-population variation. Genome. 1998;4:162-8. http://www.affymetrix.com/support/ downloads/manuals/axiom_genotyping_solution_analysis_guide.pdf. Axiom ${ }^{\circledR}$ Genotyping Solution Data Analysis Guide

5. Koch JL. Beech bark disease: the oldest "new" threat to American beech in the United States. Outlooks on Pest management; 2010. p. 64-8.

6. Houston DR (2005). Beech Bark Disease: 1934 to 2004: What's new since Ehrlich? In: Evans CA, Lucas JA and Twery MJ (2005). Beech Bark Disease: Proceedings of the Beech Bark Disease Symposium. Gen. Tech. Rep. NE-331. Newtown Square PA, US. Department of Agriculture Forest Service, Northern Research Station. 149p.

7. Houston DB, Rubin BD, Twery MJ and Steinman JR (2005). Spatial and temporal development of Beech Bark Disease in the northeastern United States. In: Evans CA, Lucas JA and Twery MJ (2005). Beech Bark Disease: Proceedings of the Beech Bark Symposium. Gen. Tech. Rep. NE-331. Newtown Square PA, US. Department of Agriculture Forest Service, Northern Research Station. 149p.

8. Castelbury LA, Rossman AY, Hyten AS. Phylogenetic relationships of Neonectria/ Cylindrocarpon on Fagus in North America. Can J Bot. 2006;84:1417-33.

9. Garnas JR, Houston DR, Ayres MP, Evans C. Disease ontogeny overshadows effect of climate and species interactions on population dynamics $n$ a nonnative forest disease complex. Ecography. 2012;35:412-21.

10. Lovett GM, Canham CD, Arthur MA, Weathers KC, Fitzhugh RD. Forest ecosystem responses to exotic pests and pathogens in eastern North America. Bioscience. 2006;56(5):395-405.

11. Ehrlich J. The beech bark disease: a Nectria disease of Fagus, following Cryptococcus fagi (Baer.). Can J Res. 1934;10(6):593-692.

12. Jakubus WJ, McLaughlin CR, Jensen PG, McNulty SA et al. (2005). Alternate year beechnut production and its influence on bear and marten populations. In: Evans CA, et al., editors. Beech Bark Disease: Proceedings of the Beech Bark Disease Symposium. Newtown Square, PA: U.S. Forest Service, Northern Research Station; 2005. Pp. 79-87.

13. McCulloough DG, Heyd RL, O'Brien JG. Biology and management of beech bark disease, Michigan's newest exotic pest. Ext. bed. E-2746. East Lansing: Michigan State University; 2001.

14. Wiggins GJ, Grant JF, Windham MT, Vance RA, Rutherford B, Klein R, Johnson K, Taylor G. Associations between causal agents of the beech bark disease complex [Cryptococcus fagisuga (Homoptera: Cryptococcidae) and Nectria spp.] in the great Smoky Mountains National Park. Environ Entomol. 2004;33(5):1274-81.

15. Wiggins GJ, Grant JF, Welbourn WC. Al-lothrombium mitchelli (Acari: Tromidiidae) in the great Smoky Mountains National Park: incidence, seasonality, and predation on beech scale (Homoptera: Ericocci-dae). Ann Entomol Soc Am. 2001;94:896-901.

16. Houston DR. A technique to artificially infest beech bark with the beech scale, Cryptococcus fagisuga (Lindinger). Res. Pap. NE-507. Broomal: U.S.
Department of Agriculture, Forest Service, Northeastern Forest Experiment Station; 1982. p. 8.

17. Houston DR (1983). American beech resistance to Cryptococcus fagisuga In Proceedings, IUFRO Beech Bark Disease Working Party Conference, 26 Sept. - 8 Oct. 1982, Hamden, Conn. USDA For. Serv. Gen. Tech. rep. WO-37.

18. Houston DB, Houston DR. Allozyme genetic diversity among Fagus grandifolia trees resistant or susceptible to beech bark disease in natural populations. Can J For Res. 2000;30:778-89.

19. Koch JL, Carey DW, Mason ME, Nelson DC. Assessment of beech scale resistance in full- and half-sibling American beech families. Can J For Res. 2010;40:265-72.

20. Mason ME, Koch JL, Krasowski M, Loo J. Comparisons of protein profiles of beech bark disease resistant and susceptible American beech (Fagus grandifolia). Proteome Sci. 2013;11:2.

21. Houston DB, Houston DR. Variation in American beech (Fagus grandifolia Ehrh.): Isozyme analysis of genetic structure in selected stands. Silvae Genetica. 1994:43:277-84.

22. Houston DR. Effect of harvesting regime on beech root sprouts and seedlings in a north-central Maine forest long affected by beech bark disease. USDA For. Serv. Northerneastern res. Newtown Square, PA, Sta. Res. Pap. NE-717.20 pp. 2001.

23. Carey DW, Kubisiak T, Houston DB, Houston DR, Koch JL (2001). RAPD analysis of post-disturbance Fagus grandifolia populations. In: Tree Biotechnology in the New Millenium, Proceedings, IUFRO Molecular Biology of Forest Trees Meeting; 2001 July 22-27; Stevenson, WA. P.169.

24. Koch JL, Carey DW (2005). The Genetics of resistance of American beech to Beech Bark disease: Knowledge through 2004. In: Evans CA, Lucas JA, Twery MJ (2005). Beech Bark Disease: Proceedings of the Beech Bark Disease Symposium. Gen. Tech. Rep. NE-331. Newtown Square PA, US. Department of Agriculture Forest Service, Northern Research Station.149p.

25. Koch JL, Carey DW. (2014). A technique to screen American beech for resistance to the beech scale insect (Cryptococcus fagisuga Lind.). J of Vis Expt. 87: doi:10.3791/51515.

26. Khan MA, Korban SS. Association mapping in forest trees and fruit crops. Exp Bot. 2012; doi:10.1093/jxb/ers105

27. Neale DB, Savolainen O. Association genetics of complex traits in conifers. Trends Plant Sci. 2004;9(7):325-30.

28. Chang S, Puryear J, Cairney J. A simple and efficient method for isolating RNA from pine trees. Plant Mol Biol Rep. 1993;11:113-6.

29. Barakat A, DiLoreto D, Zhang Y, Smith C, Baier K, Powell W, Wheeler N, Sederoff R, Carlson J. Comparison of the transcriptomes of American chestnut (Castanea dentata) and Chinese chestnut (Castanea mollissima) in response to the chestnut blight infection. BMC Plant Biol. 2009;9(1):51.

30. Gardner PP, Daub J, Tate JG, Nawrocki EP, Kolbe DL, Lindgreen S, Wilkinson AC, Finn RD, Griffiths-Jones S, Eddy SR, Bateman A. Rfam: updates to the RNA families database. Nucleic Acids Res. 2009; 37(Database issue D):136-40.

31. Koch JL, Carey DW. (2004). Controlled Cross-pollinations with American beech trees that are resistant to beech bark disease. In: Yaussy, Daniel A.; Hix, David M.; Long, Robert P.; Goebel, P. Charles, eds. Proceedings, 14th Central Hardwood Forest Conference; 2004 March 16-19; Wooster, OH. Gen. Tech. Rep. NE-316. Newtown Square, PA: U.S. Department of Agriculture, Forest Service, Northeastern Research Station: 358-364.

32. Stam (1995). JoinMap 2.0 deals with all types of plant mapping populations. Plant Genome III Abstracts.

33. Jombart T and Collins C (2015). A tutorial for Discriminant analysis of principal components (DAPC) using adegenet 2.0.0.

34. Turner SD (2014). qqman: an R package for visualizing GWAS results using Q-Q and manhattan plots. biorXiv doi: 10.1101/005165.

35. Purcell S, Chang C. PLINK v.1.9. (30 Mar 2017). http://www.cog-genomics. org/plink2.

36. Chang CC, Chow CC, Tellier LC, Vattikuti S, Purcell SM, Lee JJ. Secondgeneration PLINK: rising to the challenge of larger and richer datasets. GigaScience. 2015;4(1).

37. Barrett JC, Fry B, Maller J, Daly MJ. Haploview: analysis and visualization of LD and haplotype maps. Bionformatics. 2005;21:263-5.

38. Kahle D, Wickham H. Package 'ggmap' v. 2.6.1, Spatial Visualization with ggplot2. (23 Jan 2016). 2016. https://github.com/dkahle/ggmap.

39. Carson SD, Carson MJ. Breeding for resistance in forest trees - a quantitative genetic approach. Annu Rev Phytopathol. 1989;27:373-95. 
40. O'Malley DM. Complex trait dissection in Forest trees using molecular markers. In: BWS S, editor. The impact of plant molecular genetics. Boston: Birkhäuser; 1996.

41. Kuhlman GE, Powers HR, Pepper WD. Relative Fusiform rust resistance of loblolly and slash pine sources and families in Georgia and South Carolina. Res. Pap. SE-291. Asheville: NC: U.S. Department of Agriculture, Forest Service, Southern Research Station; 1995. p. 12.

42. Wilcox PL, Amerson HV, Kuhlman GE, Liu Ben-Hui O, O'Malley DM, Sederoff RR. Detection of a major gene for resistance to fusiform rust disease in loblolly pine by genomic mapping. Proc Natl Acad Sci U S A. 1996;93:3859-64.

43. Sniezko RA. Resistance breeding against nonnative pathogens in forest trees-current successes in North America. Can J Plant Pathol. 2006;28: S270-9.

44. Sniezko RA, Danchok R, Savin DP, Liu JJ, Kegley A. Genetic resistance to white pine blister rust in limber pine (Pinus flexilis): major gene resistance in a northern population. Can J For Res. 2016;46(9):1173-1178.

45. King JN, Alfaro RI, Hansen EM (2012). Phenotypic evidence suggests a possible major gene element to weevil resistance in sitka spruce. In Sniezko RA, Yankchuck AD, Kliejunas JT, Palmieri KM, Alexander JM, Frankel SJ. Tech cords. Proceedings of the $4^{\text {th }}$ International Workshop on Genetics of Host-Parasite Interactions in Forestry: Disease and Insect Resistance in Forest Trees. Gen. Tech. Rep. PSW-GTR-240. Albany, CA. Pacific Southwest Research Station, Forest Service, U.S. Department of Agriculuture, pp 54-64.

46. Keller B, Feuillet C, Messmer M. Genetics of disease resistance. In: Mechanisms of Resistance to Plant Diseases. (Eds): Slusarenko A, Fraser RSS, van Loon LC (2000). Dordrecht: Kluwer Academic Publishers; 2000. p. 101-160.

47. Robinson NJ, Tommey AM, Kuske C, Jackson PJ. Plant metallothioneins. Biochem J. 1993:295:1-10.

48. Lane B, Kajioka E, Kennedy T. The wheat-germ Ec protein is a zinccontaining metallothionein. Biochem Cell Biol. 1987;65:1001-5.

49. Mir G, Domènech J, Huquet G, Guo W-J, Goldsbrough P, Artrian S, Molinas M. A plant type 2 metallothionein (MT) from cork tissue responds to oxidative stress. J Exp Bot. 2004;55(408):2483-93.

50. Grennan AK. Metallothioneins, a diverse protein family. Plant Physiol. 2011; 155:1750-1.

51. Hassinen VH, Tervahauta Al, Schat H, Kärenlampi SO. Plant metallothioneins metals chelators with ROS scavenging activity? Plant Biol. 2011;13:225-32.

52. Yakovlev IA, Carl-Gunnar F, Johnsen $\varnothing$, Junttila O, Skrøppa T. Analysis of gene expression during bud burst initiation in Norway spruce via ESTs from subtracted cDNA libraries. Tree Genetics and Genomes. 2006;2:39-52.

53. Leszczyszyn OI, Imam HT, Blindauer CA. Diversity and distribution of plant metallothioneins: a review of structure, properties and functions. Metallomics. 2013;5(9):1146-69.

54. Iwata H, Minamikawa MF, Kajiya-Kanegae $H$, Ishimori M, Hayashi T. Genomics-assisted breeding in fruit trees. Breed Sci. 2016;66:100-15.

55. White TL, Adams WT, Neale DB. Forest Genetics. Cambridge: CABI Publishing, CAB International; 2007.

56. Koch JL, Carey DW, Mason ME, Nelson CD, Barakat A, Carlson JE and Neale $D$ (2011). Development of molecular tools for use in beech bark disease management. In: McManus, Katherine A, Gottscalk Kurt W (eds) 2010. Proceedings of the 21st US Department of Agriculture Interagency Research Forum on Invasive Species Gen. Tech. Rep. NRS-P-75. Newtown Square, PA: US Department of Agriculture, Forest Service, Northern Research Station, pp 38-40.

57. Zheng G, Yang Y, Zhu X, Elston RC. Analysis of Genetic Association Studies. Dordrecht Heidelberg London: Springer New York; 2012.

58. Henshall JM. Validation of Genome-Wide Association Studies (GWAS) Results. In: Gondro C, van der Werf J and Hayes B (2013). Genome-Wide Association Studies and Genomic Prediction. Methods in Molecular Biology. New York City, Humana Press, Springer Science + Business Media; 2013. p. 411.

59. van Nocker S, Gardiner SE. Breeding better cultivars, faster: applications of new technologies for the rapid deployment of superior horticultural tree crops. Hortic Res. 2014;1:14022.

60. Koch JL, Mason ME, Carey DW (2012). Screening for resistance to beech bark disease: improvements and results from seedlings and grafted field selections: In: Sniezko RA, Yanchuk AD, Kliejunas JT, Palmieri KM, Alexander JM, Frankel SJ. Tech. cords. Proceedings of the $4^{\text {th }}$ International Workshop on Genetics of Host-Parasite Interactions in Forestry: Disease and Insect Resistance in Forest Trees. Gen. Tech. Rep. PSW-GTR-240. Albany, CA: Pacific Southwest Research Station, Forest Service, U.S. Department of Agriculture, pp. 196-208.

61. Pritchard JK, Przeworski M. Linkage Disequilibrium in Humans: Models and Data. Am J Hum Genet. 2001;69(1):1-14.

62. Lewontin RC. The interaction of selection and linkage. I. General considerations; heterotic models. Genetics. 1964:49(1):49.

\section{Submit your next manuscript to BioMed Central and we will help you at every step:}

- We accept pre-submission inquiries

- Our selector tool helps you to find the most relevant journal

- We provide round the clock customer support

- Convenient online submission

- Thorough peer review

- Inclusion in PubMed and all major indexing services

- Maximum visibility for your research

Submit your manuscript at www.biomedcentral.com/submit
) Biomed Central 